\title{
Change detection in a change blindness flicker paradigm
}

\section{PsySSA}

\author{
Nafisa Cassimjee* and David J. F. Maree \\ Department of Psychology, University of Pretoria, South Africa \\ e-mail: ncassim@postino.up.ac.za
}

This study explored trends in change detection within the change blindness (CB) flicker paradigm. A sample comprising 92 university students was tested for speed in change detection. A number of alternating photographic scenes with and without changes in objects were shown in a computer laboratory. There were significant differences between males and females for central and marginal interest changes on different change types, which included changes in colour, presence and location of objects. The results were used to illustrate the methodological restrictions of previous studies and to expand on theoretical explanations for this phenomenon. This study also challenged the various notions pertaining to the nature of the representations one forms when perceiving visually. The application of connectionist principles revealed the ambiguity of representational-based explanations for change detection.

* To whom correspondence should be addressed

New discoveries often challenge old belief systems. The discovery of the change blindness (CB) phenomenon challenged the notion that the visual perception system encodes detailed and accurate representations of external reality (Rensink, O'Regan $\&$ Clarke, 1997). Research into the nature of one's ability to integrate information, in spite of motion transients, has spurred interest in the individual's limited ability to identify discrepancies between images that are altered (Simons \& Levin, 1997). Simons and Levin (1998) explored CB to social encounters and found that a person did not notice when a stranger asking for directions was switched with someone else; particularly when this switch was concealed.

Over half of their participants failed to store particulars of the scene and detect a change in physical appearance, although they had conversed with the individual for a minute prior to the replacement. Investigations into the social environment indicated that in the real world, visual memory and situated perceptions may also be influenced by the mechanisms that have been uncovered in the laboratory, thus suggesting that CB is not a 'laboratory artefact' (Noë, Pessoa \& Thompson, 2000, p. 94). 
In a laboratory setting, blindness to change under specific conditions has been assessed utilising various techniques. Although the contingency of change differs, studies on CB rely on the same design: an observer is shown a scene or image, a disruptor is introduced and the altered scene is presented. The gap, saccade or flicker-contingent approach utilises a blank image or field that is interspersed between the original and altered image to simulate a saccade or eye blink.

The constantly repeating blank image generates a visible flicker that serves as a disruptor between the original image and the altered image (Grimes, 1996; Rensink et al., 1997; Simons, 2000; Simons \& Levin, 1997). A splat-contingent technique disrupts the serial sequence between the original and changed scene with an onscreen mud splash or splat (O'Regan, Rensink \& Clarke, 1999). In the flicker-contingent approach, a repeated-change method is used where the sequence of image-altered image is continued until the observer responds and the response time is recorded.

Rensink et al. (1997) investigated CB by means of the flicker technique. The slides in their experiment consisted of real-world scenes, that is, photographs of nature, objects, and people in natural and urban settings. They distinguished between central and marginal interest changes, which consisted either of changes that formed part of the central theme or the main gist of the picture or changes that were peripheral, on the basis of independent classification by observers.

Colour differences between the scenes were considered to be equivalent to featural changes and changes involving moving or disappearing objects were classified as conjunctional modifications. The original and the changed slides were shown consecutively with a brief blank slide inserted between the slides. In Rensink et al.'s (1997) investigation, an original image was shown for $240 \mathrm{~ms}$ with the grey slide interrupting this sequence for $80 \mathrm{~ms}$. The $80 \mathrm{~ms}$ interposition of the grey slide creates a flickering display.

The sequence was an original image shown twice, followed by an altered image, also shown twice. The grey slide was inserted between each image. The original image, the distractor, and the altered image (eight slides in total) appeared in a continuous sequence that was halted once the observer responded. This process was repeated with a second set of original and altered images (see 'Procedure' below).

Rensink et al. (1997) hypothesised that this brief interruption in the visual sequence makes it difficult to perceive the changes in the scenes as it disturbs our ability to detect change by disrupting the perception of transient movement. In their experiments, Rensink et al. (1997) tested various hypotheses about CB. The first experiment examined the difference between central and marginal interest changes. They found that marginal interest changes were difficult to see, taking an average of 17.1 alternations or $10.95 \mathrm{~s}$. Central interest changes took an average of 7.3 alternations before being noticed. They compared marginal and central interests within each change type, and although no values were provided, marginal interest recognition was significantly slower than central interest changes for colour, presence and location. 
There have been many and varied explanations for the CB phenomenon. One of the more striking conjectures is that one does not form as detailed internal representations of one's environment as was previously thought. The debate centres on representationalist explanations, on the one hand, and direct perception explanations, on the other. The classical representationalist, or constructionist, model of perception states that the nature of internal representations is regarded as detailed representations and representations are constructed in the mind of the perceiver by means of the information on the retina (cf. Noë et al., 2000). A detailed conscious representation corresponding to the external environment is therefore available to the perceiver (Noë et al., 2000).

The integration of visual input across disruptions and the continuous, stable and meaningful output (visual experience) that is derived from ambiguous and distorted visual input suggest that a gap exists in quality between visual input and output. In order to generate a stable visual experience, brain mechanisms appear to integrate all incoming information.

Theorists and philosophers have argued that this chasm implied an active fillingin process involving neural compensation (Noë et al., 2000). The initial visual input comprises two inverted high-resolution retinal images distorted by blind spots and blocked by blood vessels. Neural compensatory mechanisms rebuild these images utilising the detailed pictures that are encoded, and the output is a three-dimensional recreation of the initial input.

Explanations for $\mathrm{CB}$ utilising constructionist models depend on procedural anomalies rather than presentational deficiencies. Observers, for example, may store all information that is seen, but they do not compare the separate representations because the gist of the scene is unchanged (Noë et al., 2000). This is based on the consideration that the individual stores the implicit features but is not conscious of the featural changes inherent in the image.

Alternatively, observers store the original and altered images and combine elements of the two representations. The amalgamated image is, therefore, composed of features from both images and the observer is unable to detect the change because the internal referent is a novel partial representation of the two scenes (Simons, 2000).

Direct perception explanations for the difficulty in detecting change focus on the nature of the representation. Specifically, they contest the detail and stability of internal representations formed in the act of perception, since information is available externally to the observer. Detailed external information negates the need for detailed internal representations.

Simons (2000) provides an account of plausible hypotheses for CB based on the premise that a failure to detect change arises from the vague and imprecise internal representations the individual encodes. The overwriting model alludes to the process whereby the disruptor (blank screen) overwrites the initial image and the details from the initial scene, which were not abstracted, are overwritten.

Another account states that participants encode the details of the initial image but do not apply the encoding process to the changed scene. The changed scene is 
not encoded in detail and the features of the changed image are not represented. In other words, the observer's first impression of the scene is the dominant perception because the gist of the scene remains the same and the changes do not alter the categories of input that have been stored for the first image (Pani, 2000).

The 'grand illusion' hypothesis is a radical account of the direct perception hypothesis and postulates that the individual abstracts and retains nothing about the visual world; it is the external world that fulfils the role of an extended memory store (Blackmore, Brelstaff, Nelson \& Troscianko, 1995; Dennett, 1998). The common thread inherent in the above hypotheses is that observers do not construct and retain internally the details of what they see externally and that this poor abstraction curtails their ability to detect changes in images, even when the changes are substantial.

This inference from CB studies suggests that constructionist perspectives fail to account for the experience of visual continuity that essentially is believed to arise from detailed representations. By underscoring the importance of internal representations as plausible explanations for $\mathrm{CB}$, a connectionist model may provide a novel way of understanding the nature of representations and an alternative account for the CB phenomena.

Utilising the connectionist principles to explain why failure to detect change may occur, one can argue that networks (e.g. bifurcating nerve systems) process input through the interaction of probabilistic pools of neuronal modules, which function dynamically (Bienenstock \& Geman, 1995; Dell, 1982; Vaillancourt \& Newell, 2002). This conceptualisation, unlike the common sense idea of a hard-wired brain, does not impede our ability to construe cortical functions, like visual perception and concomitant behaviour, as complex manifestations of neural activity.

Connectionist principles are not derived from accurate representations of architectonic neuronal units of the human brain, but rather from the parallel processing functions of nodal communication that underlie adaptive brain mechanisms using feedback and feed forward connections (Arbib, 1995). Thus, applying these principles to a phenomenon such as CB can provide a novel account for psychological experiments on mental processes (Sharkey \& Sharkey, 1995).

Within this framework, the nature of neuronal interactions is considered to be determined by the external environment, which sets each neuronal module's action threshold and the weighted communication channels. Encoded knowledge is the constituent of the weights and learning contributes to the modification of these weights for optimal performance of tasks. Input from the external environment determines the activity level of specific neuronal modules, and the accompanying modules which have weighted links to the activated modules simultaneously increase their activity levels to be on par with the input modules and, hence, with the input itself. Nodal weight modifications occur within the parameters of encoded knowledge.

The activity of the specific modules, together with the modifications, creates an overall pattern of stimulation that translates into the neuronal system's interpretation 
of an input. The activity level of the output modules reflects the network's interpretation of the input. Input and output modules have designated activity levels, whereas other units are dependent on learning.

In this connectionist model, the units amenable to learning must develop internal impressions (encoded in the weights), which will be utilised for input-output compatibility, resulting in optimal performance on tasks (Plaut \& Shallice, 1994). The individual's limited ability to detect change has been attributed to the brain's limited ability to encode detailed internal images (Noë et al., 2000; O’Regan, 1992).

By applying the connectionist modelling principles one can hypothesise that the sparse representations of environmental stimuli would probably diminish the weight modifications and the overall interpretation of the inputs (initial image), resulting in the 'defective' encoding and reconstruction of output. The inaccurate reproduction and inability to detect gross changes suggest that the representations are fleeting creations of neural patterns. The possibility of an accurate and extensive reproduction is minimal and is dependent on the circumstances of perceptual experience, which is represented by encoded knowledge (Damasio, 1994).

The explanations discussed above for $\mathrm{CB}$ have provided an alternative to the constructionist explanation of experience of visual continuity. It focuses on the idea that one does not retain detailed images and representations of the external world because the outside world serves as an extension of memory which enables one to integrate visual output with the salient aspects encoded internally (cf. Noë et al., 2000).

However, the connectionist interpretation also challenges the CB explanation (see 'Discussion of results'). The experience of visual continuity cannot only be a function of external representation as the CB protagonists purport. People, according to Spinney (2000), incorporate only salient details about the visual (external) world, combine them with encoded representations and beliefs (internal), and produce a continuous experience.

This complex process is reflected by anatomical evidence which shows that large cortical areas with particular physiological characteristics and connective designs are the operational bases of visual function (Hof, Nimchinsky, Ungerleider \& Morrison, 1997), and many brain regions (extrastriate visual and frontal regions) show heightened activation during change detection (Huettel, Güzeldere \& McCarthy, 2001).

The integration of visual input and output via neural compensation was also determined by Kosslyn (1994), who found that the same parts of the visual cortex were activated when a person was imagining a scene and when he or she was actually seeing it, thus supporting the notion that we use both an external and an internal memory store.

In this article, theoretical and methodological arguments against prevailing CB explanations of visual phenomena are outlined. Certain confounding issues were not taken into account in CB experiments, and the validity of explanations based on these experiments can be questioned. A review of $\mathrm{CB}$ research has shown that most of the 
studies used sample sizes that ranged from 10 to 30 observers and did not incorporate confounding variables, such as gender, age, or perceptual proficiency (Aginsky \& Tarr, 2000; Grimes, 1996; Rensink et al., 1997).

In order to address the methodological arguments against CB explanations outlined above, this study explored speed in change detection utilising a large sample of male and female university students using the flicker technique. Previous classical $\mathrm{CB}$ experiments were replicated and the study was designed to investigate trends in central or marginal interest image detection, detection cycles according to feature and conjunctional change, and gender differences in these change detection trends. On a theoretical level, ideas about the nature of representation utilising connectionist principles were explored

\section{METHOD}

\section{Sample}

The study aimed for a sample size of 120 , but the realised sample consisted of 94 first-year psychology students recruited at the University of Pretoria. This was further reduced to a sample of 92 due to missing data. Students volunteered and completed a short screening questionnaire and a consent form. The tests were available in a computer laboratory and on average, a testing session comprised 10 participants with a tester appointed to each participant. Groups were also asked not to discuss the experiments and tests with their friends, to counter confounding effects.

\section{Procedure}

The flicker test consisted of the same slides used by Rensink et al. (1997). (The original images with helpful suggestions were kindly provided by Ronald Rensink.) The slides consisted of photographs of real-world scenes ranging from nature to urban images.

The original scene was displayed for $240 \mathrm{~ms}$, followed by a grey blank scene displayed for $80 \mathrm{~ms}$, followed by the altered image displayed for $240 \mathrm{~ms}$, and a repetition of this sequence. This followed the Rensink et al. (1997) sequence of A, blank, A, blank, A', blank, A', blank, where A stands for the unchanged image and A' for the changed image.

The whole sequence was repeated until a person pressed a button to stop the display. A testee was then asked to report the observed change and the tester recorded either ' $c$ ' for correct or ' $i$ ' for incorrect. For the purposes of this article, a cycle counts as a single slide change, that is, A, blank, A', blank, which was displayed for $640 \mathrm{~ms}$ or $0.64 \mathrm{~s}$, while the total sequence (A, blank, A, blank, A', blank, A', blank) equals $1280 \mathrm{~ms}$.

The $27^{\circ}$ by $18^{\circ}$ image was displayed on a computer monitor. The slides reflected both the marginal interest (MI) changes and central interest (CI) changes that Rensink 
(2000) identified. It was hypothesised that central interest changes would be noticed quicker than marginal interests. The types of change that subjects could encounter between the original and modified image included object colour, object omission or disappearance and object location changes.

The time that elapsed between the observation of the scenes and the reported change was recorded in milliseconds as response latency. The testee was exposed to six practice slides. The six practice slides were also included in the test. To counter a sequence effect in the flicker test, the order of the images was randomised for each testee.

\section{Analysis}

The analysis of this study provided basic descriptive statistical information. The distributions of the variables were examined and it was found that the distributions for a number of variables were slightly skewed. Although the distributions could be transformed to approximate a normal distribution, it was decided to apply distributionfree tests such as the Mann-Whitney U test for comparing two groups with ordinal data. The main aim of the analysis was to provide a description of trends in order to illustrate the methodological shortcomings of previous studies.

\section{Results}

The sample of 92 students consisted of 74 (80\%) females and 18 (20\%) males. The average age for each group was 19.68 years for females and 20.55 years for males.

The dependent variable was the number of cycles that elapsed before a change was detected. The first was a distinction between MI and CI. A distinction was also made between the type of change on a particular slide which, as explained above, involved colour change $(\mathrm{C})$, position or location change of an object $(\mathrm{L})$, and the presence or absence of an object (P).

The three types of changes were paired with the interest variable so that six categories were constructed, namely, marginal-colour (MC), marginal-location (ML), mar-ginal-presence (MP), central-colour (CC), central-location (CL) and central-presence $(\mathrm{CP})$.

The measurements were made in terms of the number of cycles before a change was detected. It is thus also possible to calculate reaction time, since each slide change was displayed for 0.64 seconds. For instance, the average cycle for marginal interest for females was 13.58, which is equivalent to a reaction time of 8.69 seconds.

To facilitate comparison, the mean, standard deviation and standard error of the mean is shown in Table 1. The fastest average reaction was not less than 12 cycles while the slowest was nearly 17 cycles (marginal and location for males in Table 1). The smallest standard deviation found was for marginal interest for males $(\mathrm{SD}=$ 4.13) and the largest variation took place within $\mathrm{CP}$ for both males $(\mathrm{SD}=10.33)$ and females $(\mathrm{SD}=10.92)($ see Table 1$)$. 
Tabe 1. Statistical properties of CB-variables

\begin{tabular}{|c|c|c|c|c|c|c|c|c|c|c|c|}
\hline \multirow[b]{2}{*}{ Gender } & \multicolumn{2}{|c|}{ Female } & \multirow[b]{2}{*}{ (Median) } & \multirow[b]{2}{*}{$\begin{array}{l}\text { Standard } \\
\text { Deviation }\end{array}$} & \multirow[b]{2}{*}{ Std. Error } & \multicolumn{2}{|c|}{ Male } & \multirow{2}{*}{\multicolumn{2}{|c|}{$\begin{array}{c}\text { (Median) Standard } \\
\text { Deviation }\end{array}$}} & \multirow[b]{2}{*}{$\begin{array}{l}\text { Std. Error } \\
\text { of Mean }\end{array}$} & \multirow[b]{2}{*}{ z } \\
\hline & $\mathbf{N}$ & Mean & & & & $\begin{array}{l}\mathrm{N} \\
\text { of } \mathrm{N}\end{array}$ & $\begin{array}{l}\text { Mean } \\
\text { Mean }\end{array}$ & & & & \\
\hline Marginal interest & 74 & 13.57 & $(12,53)$ & 6.08 & .71 & 18 & 15.46 & (16.78) & 4.13 & .97 & $-1.89^{*}$ \\
\hline Central interest & 74 & 13.17 & $(10.95)$ & 6.28 & .73 & 18 & 12.46 & (11.55) & 6.26 & 1.48 & -.26 \\
\hline Location & 74 & 13.42 & $(11.81)$ & 6.53 & .76 & 18 & 14.98 & (14.07) & 5.18 & 1.22 & -1.44 \\
\hline Presence & 74 & 14.10 & $(11.19)$ & 8.25 & .96 & 18 & 14.23 & $(12.55)$ & 6.00 & 1.41 & -.79 \\
\hline Colour & 74 & 12.55 & $(10.88)$ & 5.28 & .61 & 18 & 12.91 & $(10.75)$ & 6.48 & 1.53 & -.22 \\
\hline Central + Location & 74 & 12.58 & $(9.75)$ & 7.67 & .89 & 18 & 13.59 & (11.54) & 7.27 & 1.71 & -.69 \\
\hline Central + Presence & 74 & 14.22 & $(10.68)$ & 10.92 & 1.27 & 18 & 12.41 & $(9.75)$ & 10.33 & 2.44 & -1.05 \\
\hline Central + Colour & 74 & 12.54 & $(11.42)$ & 7.28 & .85 & 18 & 12.20 & (9.88) & 7.86 & 1.85 & -.28 \\
\hline Marginal + Location & 74 & 14.24 & $(11.88)$ & 8.81 & 1.02 & 18 & 16.78 & $(14.50)$ & 8.74 & 2.06 & -1.31 \\
\hline Marginal + Presence & 74 & 13.74 & $(10.88)$ & 9.64 & 1.12 & 18 & 16.15 & (13.77) & 8.89 & 2.09 & $-1.65^{*}$ \\
\hline Marginal + Colour & 74 & 12.56 & $(11.38)$ & 6.58 & .80 & 18 & 13.61 & $(10.16)$ & 9.82 & 2.31 & -.13 \\
\hline AGE & 74 & 19.69 & $(19.00)$ & 1.53 & .18 & 18 & 20.56 & (19.00) & 3.33 & .78 & \\
\hline
\end{tabular}

$* \mathrm{p}<0.05$ (one-tailed) ** Age indicated in years and all the other scores indicated in number of sequences i.e. A, blank, A', blank. One sequence equals $640 \mathrm{~ms}$

The comparisons are presented in Figures 1 to 4 in order to illustrate the trends, direction of differences and magnitude of differences. Figure 1 shows the difference between males and females for interest. It is clear that males were slower than females on marginal interest, while the reverse is true for central interest. Using the Mann-Whitney $\mathrm{U}$ test, a significant difference was found between males and females for marginal interest $(z=1.885, p<0.05)$. Males were significantly slower.

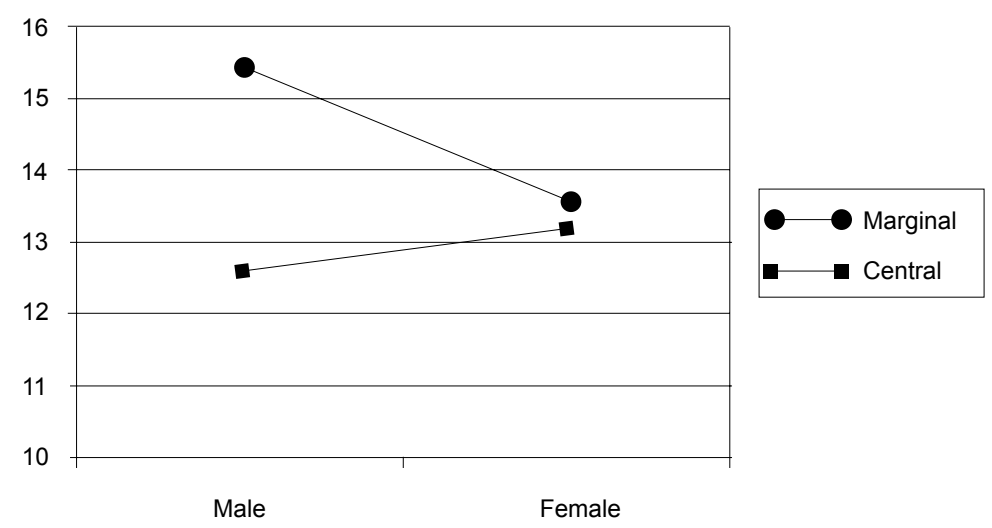

Figure 1. Comparison between genders for interest 
Nafisa Cassimjee and David J. F. Maree

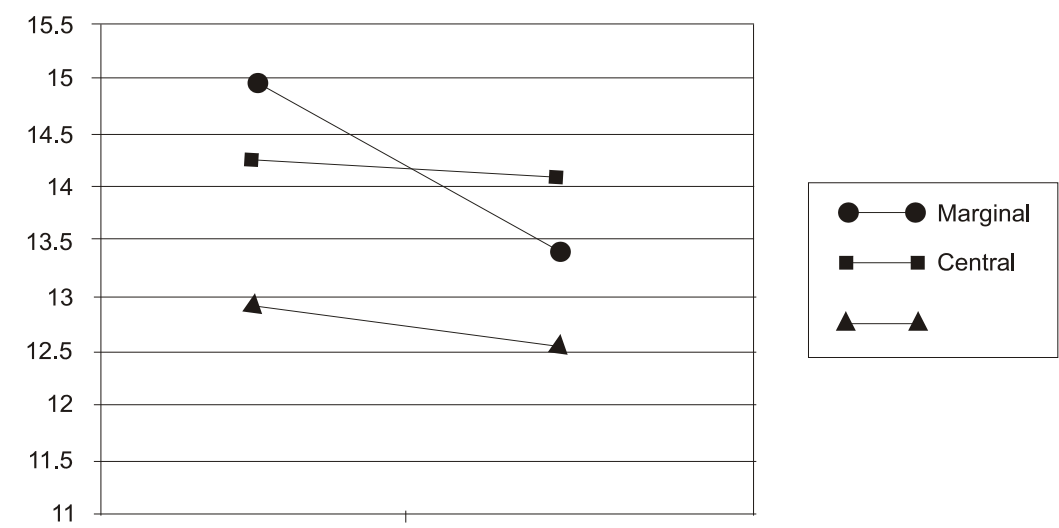

Figure 2. Comparison between genders for type of change

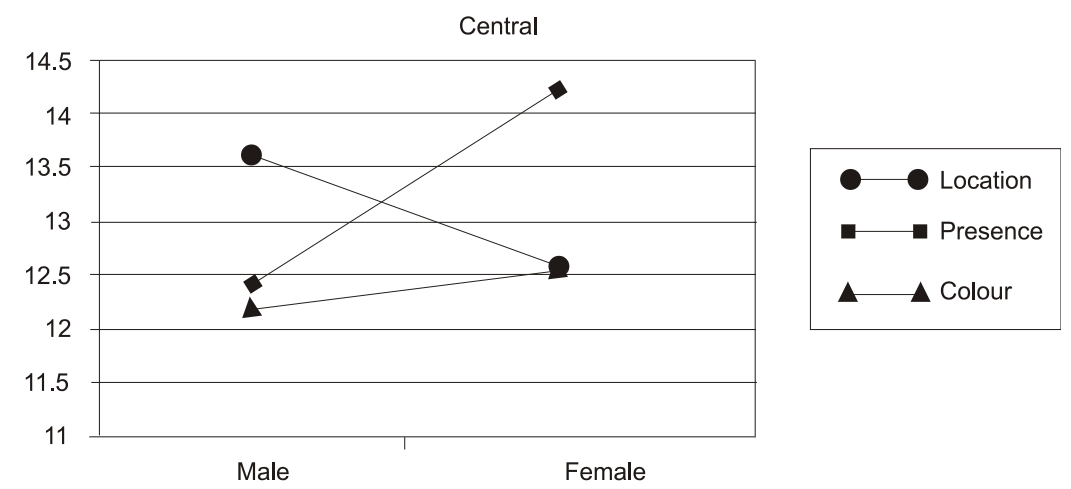

Figure 3. Comparison between genders for central interest and type of change


Figure 4. Comparison between genders for marginal interest and type of change 
According to Figure 2, the males performed consistently slower than females. A sharper drop in performance was found for location. No significant differences were found between males and females for change type. Figure 3 shows the average speed for change type within the category central interest. It is interesting to note that males performed slower than females for location, but not for presence and colour.

Thus, the faster average performance for males found on central interest (Figure 1) concealed the reverse situation with location. It is also interesting to see that the detection of presence and colour for central interest was quicker for males. However, the differences between males and females were not significant. The faster performance by females for change type was consistent in the category, marginal interest, as shown in Figure 4. Females performed significantly faster than males on presence for marginal interest $(z=1.654, p<0.05)$.

\section{DISCUSSION}

This study duplicated previous research, such as that of Rensink et al. (1997), with the main difference being the sample size. Previous studies had smaller samples, which have the effect of highlighting the peculiarities of the individuals within the sample. Selecting a random sample, whilst being aware of the characteristics of the sample and the population from which it was drawn, minimises the role of confounding variables.

However, more often than not, where basic research on perceptual abilities is concerned, one may easily assume that the characteristics necessary for the functioning of the visual system should remain invariant across individuals and groups. Researchers could hastily generalise findings and conclusions with regard to the phenomena under study. The authors do not pretend that this was the case with $\mathrm{CB}$ research. They are attempting to investigate the mechanisms, and in the process, avoid falling into obvious methodological traps.

Rensink et al. (1997) and other researchers who had conducted related studies, did not describe their samples in terms of gender, age or other possible confounding variables. The reaction times found in this study are comparable to those reported by Rensink et al. (1997). The single feature changes (colour), which are processed in a parallel manner, were more easily detected than conjunctional changes (presence and location), which are processed sequentially (Grossberg, 1999).

The slower detection time for the former is attributable to the consecutive processing mechanisms involving the detection, encoding and recall of accurate configurations within and between objects and comparison of different configurations. Featural changes, on the other hand, involve parallel processing mechanisms that compare the colours of overlapping components without the object of the scene being analysed or recognised. In terms of detection speed, featural changes require minimum processing and observers register faster reaction times.

In contradistinction to the results of Rensink et al. (1997), no difference in marginal and central interest categorisation of slides was found. However, as soon as 
the sample was divided into males and females, similar results to that of Rensink et al. (1997) were found; that is, a significant difference between central and marginal interest was apparent. However, it is interesting to note that this significant result was confined to males. Males in the current sample saw changes in marginal interest slides within an average of 15.46 cycles, while that for central interest was 12.64 . No difference in reaction speed for females was found. The trend that females detected change slightly faster than males when change-type (colour, location and presence) is concerned was reflected by a similar trend within marginal interest.

The trend was reversed within the central interest category. Males detected change within colour and presence faster than females. Within the central interest category, females detected only the location change-type faster. These trends may indicate the prominent effect of central interest on males' ability to detect changes when presence and colour are concerned but it also indicates the stability of females' ability to detect when an object in the visual environment has changed location.

The current study maintains that whatever the explanation for the findings may be, the size of the sample and the characteristics of the sample can hide significant aspects related to CB. It is a tendency with research on basic cognitive and perceptual functions to utilise small samples, and even to discount the sample characteristics. However, certain characteristics may confound the findings, thereby invalidating the attempts to explain cognitive and perceptual phenomena.

The question remains, over and above the observations made in respect of methodology, what are the implications of the findings of the current study? With regard to the gender differences in reaction speed as it pertains to marginal and central interest, one can speculate that it reflects the different cognitive and perceptual strategies employed by females and males.

In sum, the findings of the present study were that females were quicker to locate differences than males in marginal interest images, especially when an object is present or not (presence). No significant differences in speed of change detection were found for central interest images, that is, obvious objects changing location and colour or disappearing.

Although the authors do not want to belabour the point on gender differences, one is reminded of research done by Kimura (1992), which showed that women outperform men in accuracy ratings on object location and perceptual speed, whereas men perform better at spatial rotation and target location.

These trends were reiterated by Weiss, Kemmler, Deisenhammer, Fleischhacker and Delazer's (2003) short overview on cognitive and ability differences between males and females. Previous research showed that males tend to perform better on spatial tasks, mathematical reasoning, route navigation and target-directed motor skills (cf. Lim, 1994). However, females perform better in linguistic tasks, fine eye-hand coordination tasks, and perceptual speed tasks, such as rapidly identifying matching items.

The trend in the better performance of females on perceptual speed is confirmed by Kimura's (1999) recent overview on gender-based cognitive differences. 
Furthermore, neuropsychological lesion studies have indicated that the left hemisphere is dominant for processes specific to imagery generation, and in males specific visio-spatial processes are laterialised to the right hemisphere (Behrmann, 2000).

Current explanations for CB support the direct perception hypothesis as opposed to constructionist, or top-down processing, models and are cited as evidence for the theory that the brain does not store detailed internal representations. However, a connectionist interpretation can yield conflicting explanations for change detection and the nature of internal representations. In the CB experiment, the gist of the scene remained the same although there were colour, location and object changes in the second image. In connectionist terms, if the gist of the scene remains the same, the neural connectionist networks respond from the same state space (activity level) without updating the pattern of activity and the parameters of the attractor basin (final pattern), which was determined by the neural pattern set when an individual viewed the first image.

Attractors function according to the principle of similarity where the same output is mapped for similar inputs (Plaut \& Shallice, 1994). The key element to updating the attractor and the attractor basin in change detection is focused visual attention, which is subserved by an extensive network of brain areas (Huettel et al., 2001).

When an individual finally observes the change, the connection weights modulate the interaction between units and the attractor basin is rebuilt in state space. Being aware of the changes institutes a type of learning which acts on the weight modulators and on the neural representations, resulting in the final activity pattern being mapped onto the initial pattern of activity (Hanson \& Burr, 1990).

Change detection from this perspective is thus a slow process (which was shown in the results above) - it does not happen instantaneously. Moreover, change detection has been associated with activity in many brain regions that are complementary in nature, and neurological processes occurring in many brain regions may underscore the slow response times associated with detecting change.

The implication of a sparse internal representation (reflected by the direct perception hypothesis) is a slow detection of change based on an outdated attractor pattern that represents the initial image. No updated neural pattern can be mapped on an already existing activity level. However, if a detailed internal representation is present, then detection of change will also be slow because an activity level has been set and the attractor basin allows for interpretations of input that are based on similarity and the response will be based on the activity pattern set for the initial image.

Despite the clear-cut explanations provided for the change detection phenomenon, connectionist principles indicate that focusing on the nature of representations may be premature, since slow processing can be explained by either detailed or sparse representations. As there is evidence from neuropsychological studies that information can be represented and processed outside of conscious awareness, it is thus probable that an observer's visual representational ability may not underlie the modest performance on change detection tasks. This hypothesis is consistent with the 
research findings, which indicate that participants may be aware of changes but unable to report them (Fernandez-Duque \& Thornton, 2000; Hayhoe, 2000).

This contention supports the idea of delineated visual processing streams and dissociation between two modes of visual processing (Bridgeman, 1995). The cognitive/ventral (pattern recognition, object position) and sensorimotor/dorsal (quantitative spatial information, visually guided behaviour, i.e. location and motion) representations can be mapped onto distinct anatomical pathways in the occipito-parietal and occipito-temporal areas, respectively.

The neuropsychological condition of blindsight provides a heuristic for understanding how the two processing streams function. The assumption is that blindsight patients, who demonstrate a lack of awareness of object position, yet are able to accurately point out its location, have processing anomalies in the cognitive, but not the sensorimotor, systems. In other words, among blindsight patients there is accurate visually guided behaviour without perceptual experience or cognitive awareness.

Unlike the patients with blindsight, participants in CB experiments report an awareness of change, but an inability to report the position of the change or to visually guide behaviour. Perhaps during displacement (intermittent grey slides), the flow of perceptual information is disrupted and the sensorimotor system cannot adequately access the higher perceptual processes of knowing (Zillmer \& Spiers, 2001).

There is perceptual awareness in the absence of accurate positioning information and visually guided behaviour. Furthermore, the sensorimotor system has no memory, and in a change detection task, relies on the information from the cognitive system in order to report change (Bridgeman, 1995). The flicker technique uses motion to interrupt the two images, and this corroborates the hypothesis that the limited ability to detect change can be a function of disrupted motion systems, and not necessarily of cognitive or representational systems only. The lack of perceptual reports therefore, may lead to an erroneous underestimation of the extent of internal representations (Hayhoe, 2000).

In conclusion, the results have shown that $\mathrm{CB}$ cannot be explained unequivocally. Various ideas on the nature of the representations one forms when visually perceiving have been posed and briefly alluded to. This study proposed that the nature of representations is not sufficient to explain the phenomenon of CB.

Arguments were proposed on two levels, namely, theoretical and methodological. Theoretically, connectionism provides alternative but conflicting explanations on the nature of representations that underscore the speed at which one detects change. This implies, along with evidence from other sources, that change detection could be a function of different neural probabilities, which are not necessarily confined to cognitive and representational systems.

Significant gender differences were reported for marginal and central interest changes pertaining to certain change types. This is important when investigations of change detection measure response latency and attention response latency. Researchers are only now beginning to suspect that factors over and above attention or basic 
information processing mechanisms are responsible for the speed of change detection (Hollingworth \& Henderson, 2000). If the semantic content of a change mediates its detection speed, aspects such as perceptual style and stimulus dependency/independency could play a role in change detection speed. The construct of interest appears to be an important gender specific factor in the detection of change. On a methodological level, disregarding confounding effects such as gender could limit the scope for explanations of $\mathrm{CB}$.

\section{REFERENCES}

Aginsky, V. and Tarr, M.J. (2000). How are different properties of a scene encoded in visual memory? Visual Cognition, 7, 147-162.

Arbib, M.A. (1995) (Ed.). The handbook of brain theory and neural networks. Cambridge: MIT.

Behrmann, M. (2000). The minds eye mapped onto the brain's matter. Current Directions in Psychological Science, 9(2), 50-54.

Blackmore, S.J., Brelstaff, G., Nelson, K. and Troscianko, T. (1995). Is the richness of our visual world an illusion? Transsaccadic memory for complex scenes. Perception, 24, 1075-1081.

Bienenstock, E. and Geman, S. (1995). Compositionality in neural systems. In M.A. Arbib (Ed.), The handbook of brain theory and neural networks (pp. 223-226). Cambridge: MIT.

Bridgeman, B. (1995). Dissociations between visual processing modes. In M.A. Arbib (Ed.), The handbook of brain theory and neural networks (pp. 318-320). Cambridge: MIT.

Damasio, A.R. (1994). Descartes' error: Emotion, reason, and the human brain. New York: Putnam.

Dell, P.F. (1982). Beyond Homeostasis: Toward a concept of coherence. Family Process, 21, 21-41.

Dennett, D.C. (1998). No bridge over the stream of consciousness. Behavioural and Brain Sciences, 21, 753-754.

Fernandez-Duque D. and Thornton, I.M. (2000). Change detection without awareness: Do explicit reports underestimate the representation of change in the visual system? Visual Cognition, 7, 323-344.

Grimes, J. (1996). On the failure to detect changes in scenes across saccades. In K. Atkins (Ed.), Perception (pp.89-110). New York: Oxford Press.

Grossberg, S. (1999). The link between brain, learning, attention, and consciousness. Consciousness and Cognition, 8, 1-44.

Hanson, S.J. and Burr, D.J. (1990). What connectionist models learn: Learning and representation in connectionist networks. Behavioural and Brain Sciences, 13, 471-518.

Hayhoe, M. (2000). Vision using routines: A functional account of vision. Visual Cognition, 7, 43-64

Hof, P.R., Nimchinsky, E.A., Ungerleider, L.G. and Morrison, J.H. (1997). Morphologic and neurochemical characteristics of corticocortical projections: Emergence of circuit specific 
features and relationships to degenerative changes in Alzheimer's disease. In B.T. Hyman, C. Duyckaerts and Y. Christen (Eds), Connections, cognitions and Alzheimer's disease (pp. 59-82). Berlin: Springer.

Hollingworth, A. and Henderson, J.M. (2000). Semantic informativeness mediates the detection of changes in natural scenes. Visual Cognition, 7(1-3), 213-235.

Huettel, S.A., Güzeldere, G. and McCarthy, G. (2001). Dissociating the neural mechanisms of visual attention in change detection using functional MRI. Journal of Cognitive Neuroscience, 13(7), 1006-1018.

Kimura, D. (1992, September). Sex differences in the brain. Scientific America: Mind and Brain, 81-87.

-. (1999). Sex and cognition. Cambridge, MA: MIT Press.

Kosslyn, S.M. (1994). Image and the brain. Cambridge: MIT Press.

Lim, T.K. (1994). Gender-Related Differences in Intelligence: Application of Confirmatory Factor Analysis. Intelligence, 19, 179-192.

Noë, A., Pessoa, L. and Thompson, E. (2000). Beyond the grand illusion: What CB really teaches us about vision. Visual Cognition, 7, 93-106.

O' Regan, J.K. (1992). Solving the "real" mysteries of visual perception: The world as an outside memory. Canadian Journal of Psychology, 46, 461-488.

O' Regan J.K., Rensink, R.A. and Clarke, J.J. (1999). CB as a result of 'mudsplashes'. Nature, $398,34$.

Pani, J.R. (2000). Cognitive description and CB. Visual Cognition, 7, 107-126.

Plaut, D. and Shallice, T. (1994). Connectionist modelling in cognitive neuropsychology: A case study. London: Lawrence Erlbaum.

Rensink, R.A. (2000). When good observers go bad: CB, inattentional blindness, and visual experience. Psyche, 6(9), 87-95.

Rensink, R.A., O’Regan, J.K. and Clarke, J.J. (1997). To see or not to see: The need for attention to perceive changes in scenes. Psychological Science, 8, 368-373.

Sharkey, A.J.C. and Sharkey, N.E. (1995). Cognitive modelling: Psychology and connectionism. In M.A. Arbib (Ed.), The handbook of brain theory and neural networks (pp. 200-203). Cambridge: MIT.

Simons, D.J. (2000). Current approaches to CB. Visual Cognition, 7,1-15.

Simons, D.J. and Levin, D.T. (1997). CB. Trends in Cognitive Sciences, 1, 261-267.

- (1998). Failure to detect changes to people during real-world interaction. Psychonomic Bulletin and Review, 4, 644.

Spinney, L. (2000). Blind to change. New Scientist, 2265, 29-32.

Vaillancourt, D.E. and Newell, K.M. (2002). Changing complexity in human behavior and physiology through ageing and disease. Neurobiology of Ageing, 23, 1-11.

Weiss, E.M., Kemmler, G., Deisenhammer, E.A., Fleischhacker, W.W. and Delazer, M. (2003). Sex differences in cognitive functions. Personality and Individual Differences, 35(4), 863-875.

Zillmer, E.A. and Spiers, M.V. (2001). Principles of Neuropsychology. Belmont: Wadsworth. 